\begin{tabular}{|l|}
\hline Encyclopedia of Earth Sciences Series \\
\hline Encyclopedia of Solid Earth Geophysics \\
\hline Springer Science+Business Media B.V. 2011 \\
\hline $10.1007 / 978-90-481-8702-7 \_115$ \\
\hline Harsh K. Gupta \\
\hline
\end{tabular}

\title{
Geomagnetic Field, Global Pattern
}

\author{
Susan Macmillan ${ }^{1}$ \\ (1) British Geological Survey Murchison House, West Mains Road, EH9 3LA Edinburgh, Scotland, UK \\ $\triangle$ Susan Macmillan \\ Email: smac@bgs.ac.uk
}

\section{Without Abstract}

\section{Introduction}

The geomagnetic field is generated in the fluid outer core region of the Earth by electrical currents flowing in the slowly moving molten iron. In addition to sources in the Earth's core, the geomagnetic field observable on the Earth's surface has sources in the crust and in the ionosphere and magnetosphere. The signal from the core dominates, accounting for over $95 \%$ of the field at the Earth's surface. The geomagnetic field varies on a range of scales, both temporal and spatial; the description of the variations made here concentrates on the recent spatial and temporal variations of the field with origins in the Earth's core that can be surmised from observations made over the last four centuries.

\section{Observations}

The geomagnetic field is a vector quantity, having magnitude as well as direction. The direction of the geomagnetic field has been directly observed and recorded for at least 400 years beginning with observations of the angle in the horizontal plane between true north and the magnetic field vector, known as declination or magnetic variation. In 1634, it was realized by Henry Gellibrand that declination in London was changing with time when he compared his observations with those made by Edmund Gunter 12 years earlier. Since then, this important discovery of secular variation has ensured regular observations of the magnetic field through time. Measurements of declination were important for navigation across the oceans, and a source of early observations is ships' logbooks (Jackson et al., 2000). Before long it was also realized that other elements of the geomagnetic field were of interest, in particular the angle of dip of the magnetic field vector from the horizontal known as inclination, and this was also measured. However, it was not till 1832 when Carl Freidrich Gauss established his technique for measuring absolute intensity did we have accurate measurements of the magnitude of the geomagnetic field.

As demands on accuracy and interest in the geomagnetic field increased, permanent observatories began to be established. Since the 1840 s the number of observatories around the world has slowly increased but has now dropped to about 160 in recent times. The advent of the proton precession magnetometer and the fluxgate magnetometer in the twentieth century considerably eased the automation of observatories during this time. However, regular manual absolute observations, nowadays of declination and inclination using a fluxgate theodolite, are necessary to maintain accurate baseline control over long periods of time. There are also networks of repeat stations providing data over extensive areas from which country-wide magnetic charts can be derived. More recently, magnetic measurements made by satellites have become important in determining the pattern of the geomagnetic field. In particular, vector data from Magsat (1979-1980), Ørsted (1999-current), and CHAMP (2000-2010) have all been utilized in the production of recent spherical harmonic models of the geomagnetic field.

\section{Spherical harmonic analysis}

Till the 1950s, magnetic charts at a given epoch for use in navigation depended on manually drawing contours through the observations (Malin, 1971). Although spherical harmonic analysis had been developed in 1839 by Carl Freidrich Gauss, it was not routinely used to fit mathematical models to the observations till the advent of computers. Before computers 
spherical harmonic analysis required that the data be in the form of values of one or more of the orthogonal components $X$, $Y$, and $Z$ of the geomagnetic field (magnetic intensities in the northerly, easterly, and vertically down directions) at points regularly spaced in latitude and longitude (Barraclough, 1978). This involved the intermediate stage of interpolating values from manually drawn contour charts.

The mathematical and physical basis for spherical harmonic analysis is now given. In a source-free region, the Earth's magnetic field $B$ is the negative gradient of a magnetic potential $V$ that satisfies Laplace's equation:

$$
B=-\nabla V \text { where } \nabla^{2} V=0
$$

A solution to Laplace's equation for the magnetic potential arising from sources inside the Earth at a given epoch is given by:

$$
\begin{aligned}
V(r, \theta, \lambda)= & a \sum_{n=1}^{n_{\operatorname{mar}}}\left(\frac{a}{r}\right)^{n+1} \\
& \sum_{m=0}^{n}\left(g_{n}^{m} \cos m \lambda+h_{n}^{m} \sin m \lambda\right) P_{n}^{m}(\theta)
\end{aligned}
$$

In this equation, $r, \theta, \lambda$ are geocentric coordinates ( $r$ is the distance from the center of the Earth, $\theta$ is the colatitude, i.e., $90^{\circ}$ - latitude, and $\lambda$ is the longitude), $a$ is a reference radius for the Earth $(6371.2 \mathrm{~km}), g_{n}^{m}$ and $h_{n}^{m}$ are the spherical harmonic Gauss coefficients of degree $n$ and order $m, P_{n}^{m}(\theta)$ are the Schmidt semi-normalized Legendre functions and $n_{\max }$ is the maximum degree of the spherical harmonic expansion. If the observations of the magnetic field are spread over time, the magnetic potential and corresponding Gauss coefficients are also dependent on time with splines being commonly used.

\section{Maps of the geomagnetic field}

It is worth noting here that the first published map of the geomagnetic field is that of declination made in 1701 by Edmond Halley. It was based on declination observations made during a magnetic survey expedition under naval command and covered the Atlantic Ocean (Clark and Barraclough, 2001).

The maps that we show here are based on spherical harmonic models. Nowadays, there are many such models of the geomagnetic field and here we look at two examples. One is the gufml model which is derived from four centuries of magnetic field observations (Jackson et al., 2000) and extends to spherical harmonic degree 14 with B-splines being used to fit the variations in time from 1590 to 1990 . It has smoothness imposed in both the spatial and temporal domains. The other model is the International Geomagnetic Reference Field (Macmillan and Finlay, 2011) produced under the auspices of the International Association of Geomagnetism and Aeronomy (IAGA). It extends to spherical harmonic degree 10 till 1995 and thereafter extends to degree 13. The variations in time from 1900 to 2015 in the IGRF are assumed to be piecewise linear and there is effectively no smoothness imposed in either the spatial or temporal domains. The final IGRF coefficients are made up of weighted averages of candidate sets of coefficients from (mostly) unregularized models.

Using the gufm 1 model, plots of declination and inclination at 1600, declination, inclination, and total intensity at 1950 are shown in Figures 1 and 2 . Using the eleventh generation IGRF, plots of the magnetic elements at 2010 and their rates of change are shown in Figures 3 - -9 . The rates of change plots are derived from a predictive secular variation model which extends to spherical harmonic degree 8. Up-to-date maps, and on-line calculators are available from a number of websites, for example, http://www.geomag.bgs.ac.uk/navigation.html and http://www.ngdc.noaa.gov/geomag /WMM/DoDWMM.shtml.
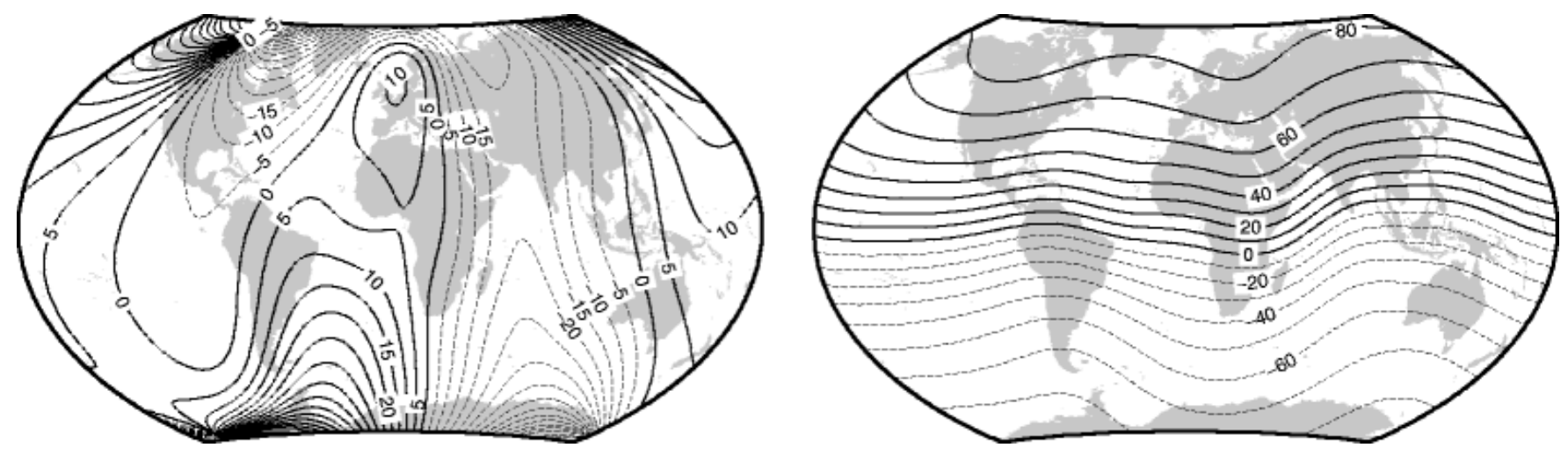

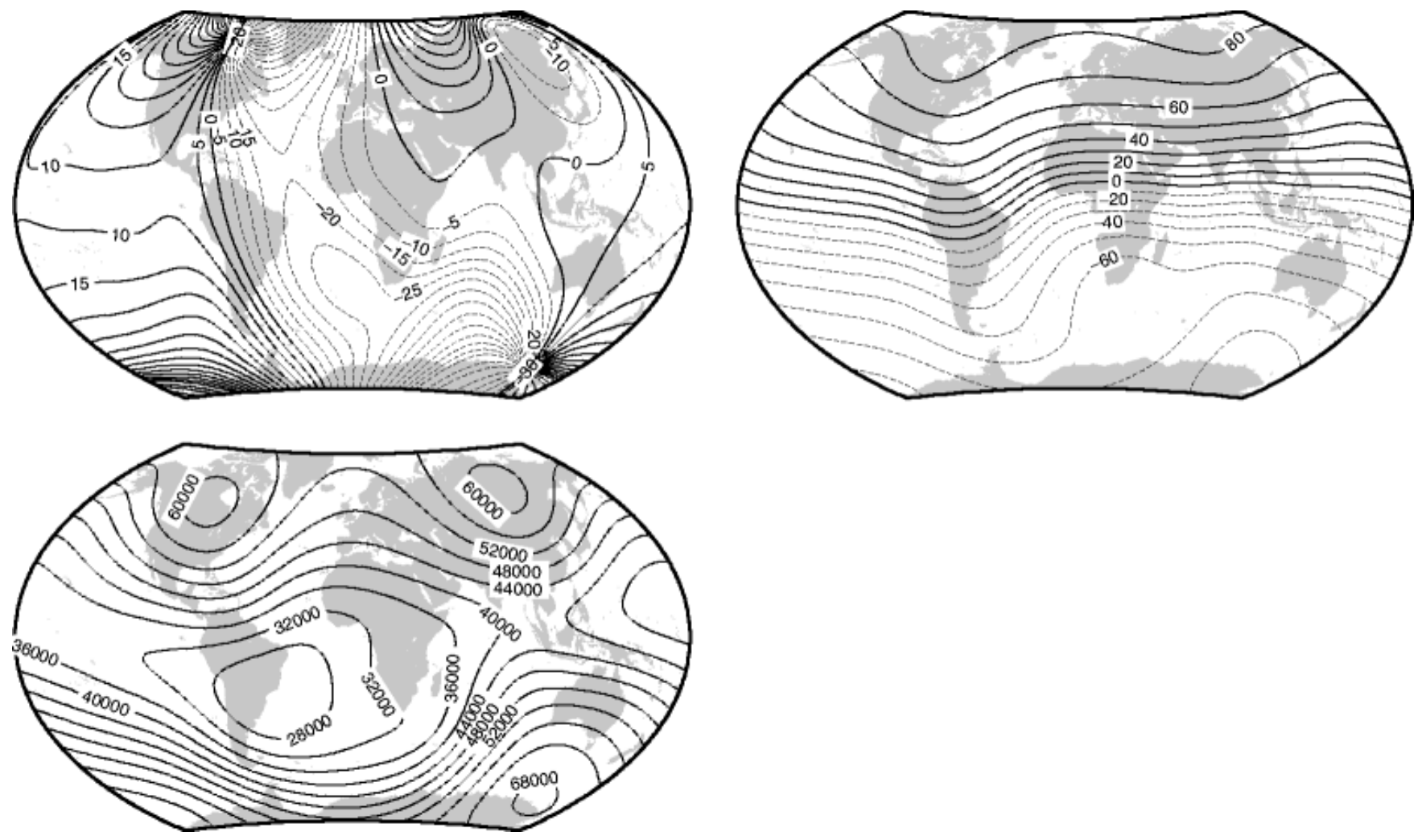

Geomagnetic Field, Global Pattern, Figure 2 Declination, inclination (degrees), and total intensity (nT) at 1950.0 computed from the gufm1 model.
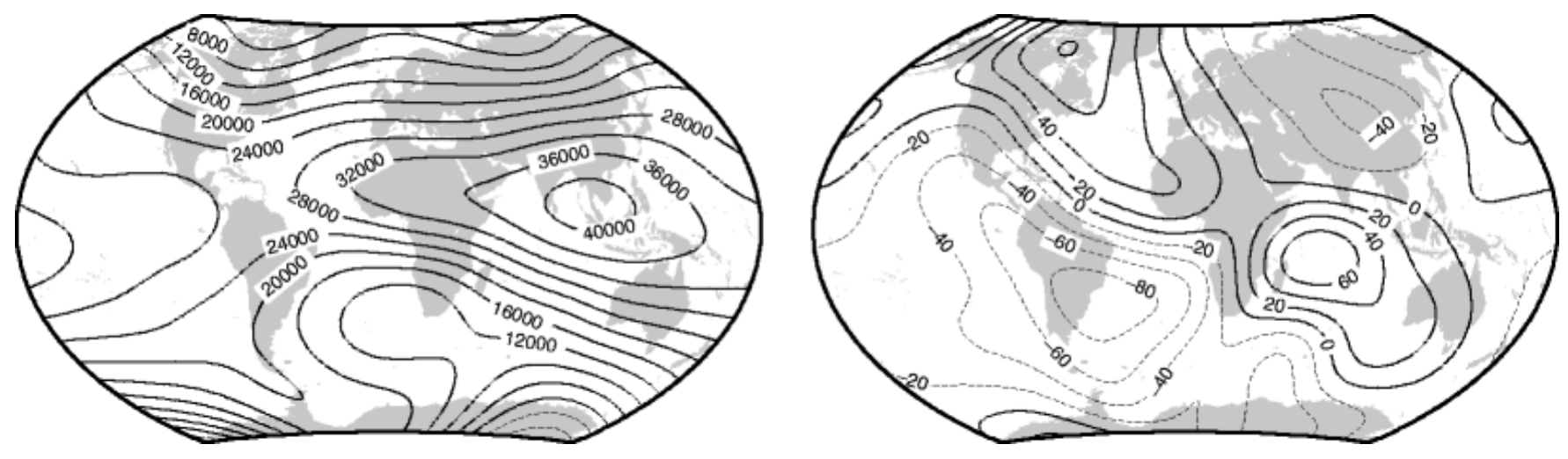

Geomagnetic Field, Global Pattern, Figure 3 Northerly intensity (nT) at 2010.0 and its rate of change (nT/year) for 2010.0-2015.0 computed from IGRF-11.
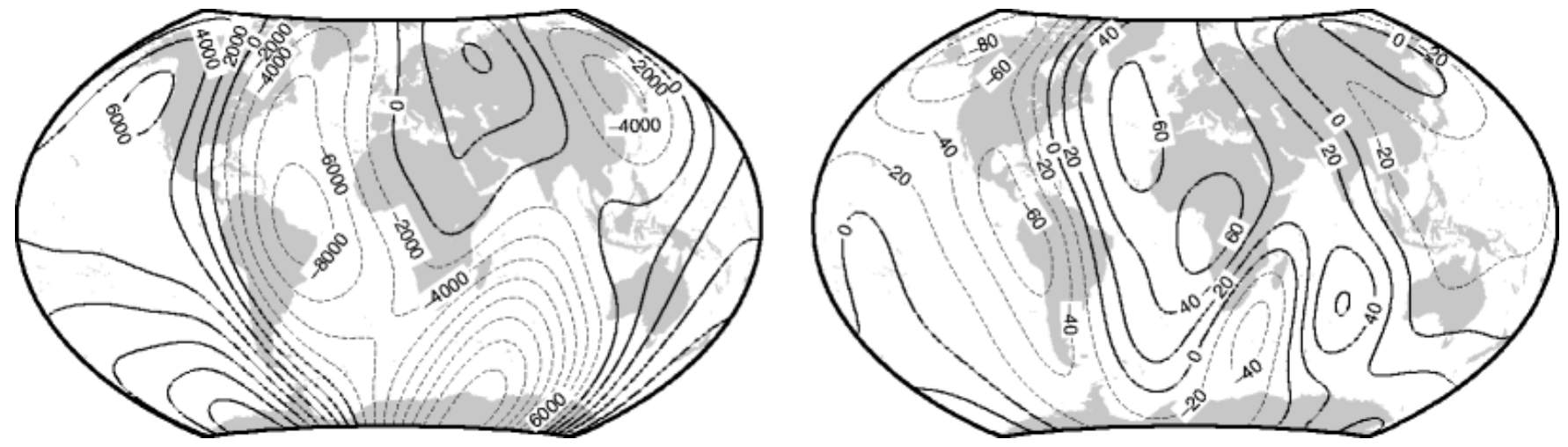

Geomagnetic Field, Global Pattern, Figure 4 Easterly intensity (nT) at 2010.0 and its rate of change (nT/year) for 2010.0-2015.0 computed from IGRF-11. 

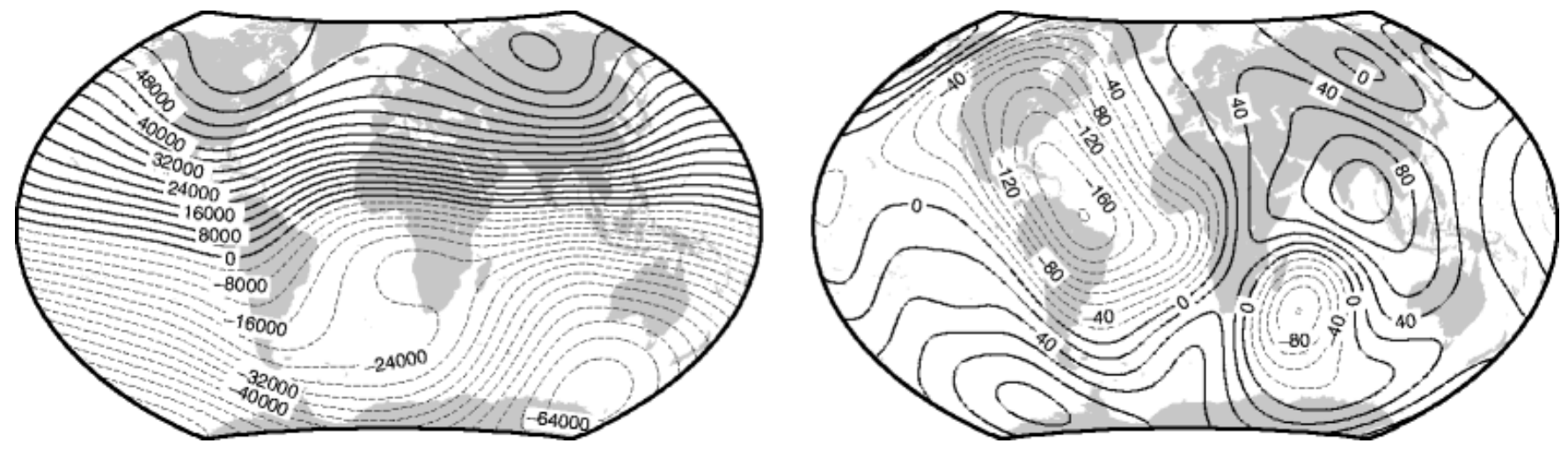

Geomagnetic Field, Global Pattern, Figure 5 Vertical intensity (nT) at 2010.0 and its rate of change (nT/year) for 2010.0-2015.0 computed from IGRF-11.
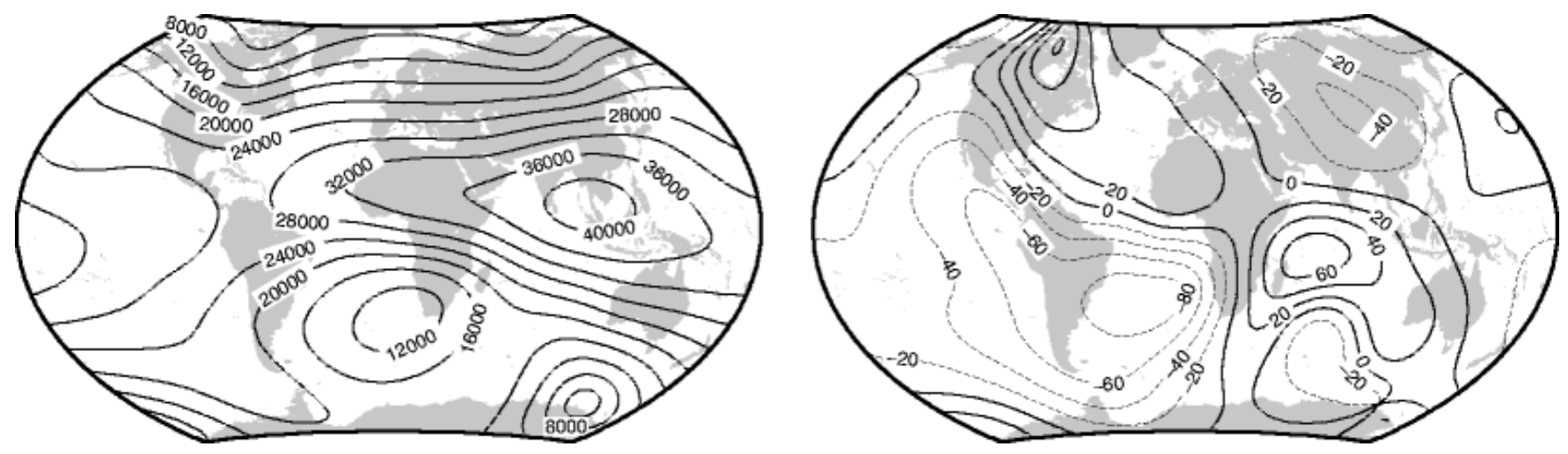

Geomagnetic Field, Global Pattern, Figure 6 Horizontal intensity (nT) at 2010.0 and its rate of change (nT/year) for 2010.0-2015.0 computed from IGRF-11.
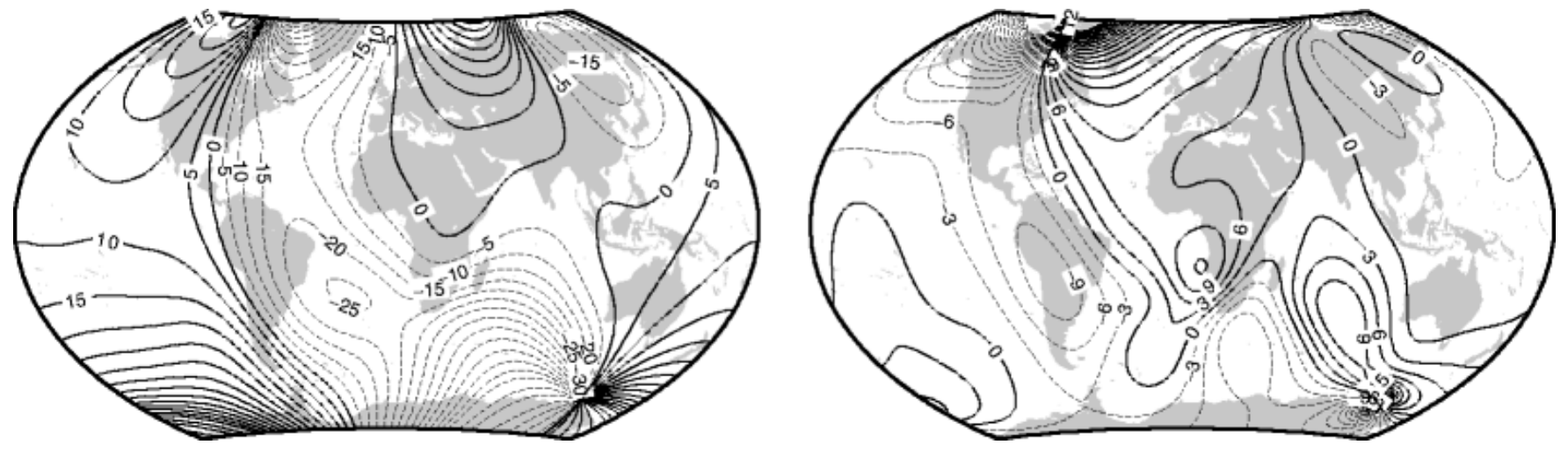

Geomagnetic Field, Global Pattern, Figure 7 Declination (degrees) at 2010.0 and its rate of change (arc-minutes/year) for 2010.0-2015.0 computed from IGRF-11.
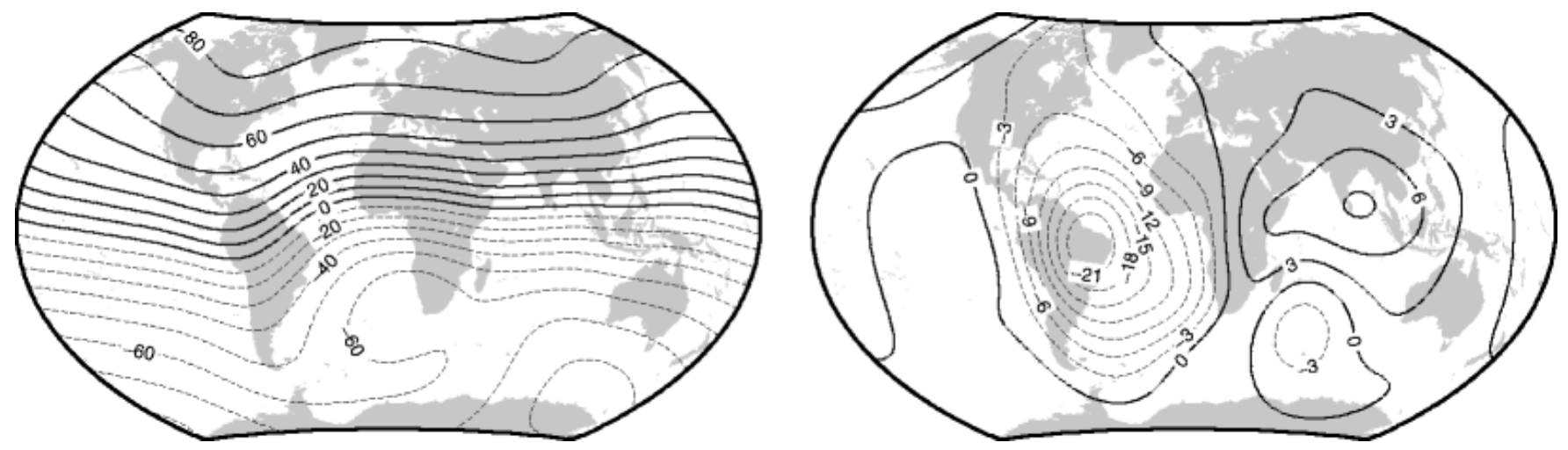
Geomagnetic Field, Global Pattern, Figure 8 Inclination (degrees) at 2010.0 and its rate of change (arc-minutes/year) for 2010.0-2015.0 computed from IGRF-11.
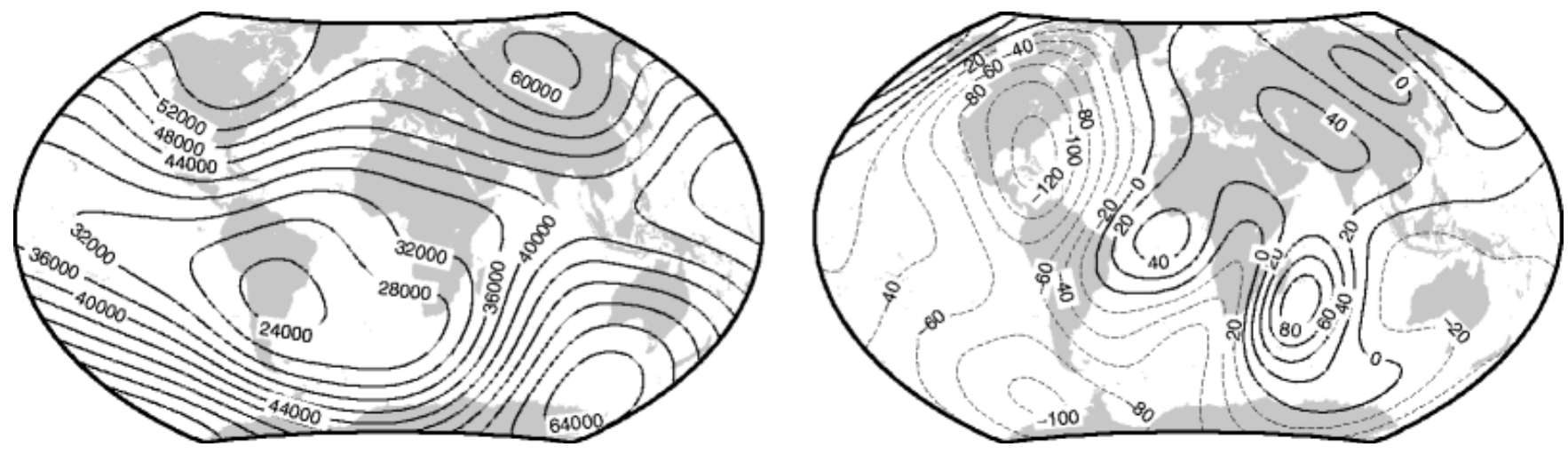

Geomagnetic Field, Global Pattern, Figure 9 Total intensity (nT) at 2010.0 and its rate of change (nT/year) for 2010.0-2015.0 computed from IGRF 11.

Features of note on these maps are the dip equator and dip poles in the maps of inclination (Figures $\underline{1}, \underline{2}$ and $\underline{8}$ ), and the area of weak magnetic field in the South Atlantic, and strong magnetic field near the poles in Figures 2 and $\underline{9}$. The dip equator is where the magnetic field is horizontal (inclination $=0^{\circ}$ ) and a current system is set up in the upper atmosphere called the equatorial electrojet. The dip poles (also called magnetic poles) are locations where the magnetic field is, on average, vertical. Another set of poles is the dipole poles or geomagnetic poles. Their positions can be derived from the degree 1 spherical harmonic coefficients $\left(g_{1}^{0}, g_{1}^{1}\right.$, and $\left.h_{1}^{1}\right)$. Associated with the geomagnetic poles, in approximately oval-shaped loci, are the auroral electrojets in the upper atmosphere. The electrojets, particularly the auroral electrojets, generate rapid time-varying magnetic fields of significant amplitude.

\section{Recent changes in the geomagnetic field}

From a comparison of the declination maps in Figures $\underline{2}$ and $\underline{7}$ it can be seen that the zero contour (the agonic line) is moving slowly westwards with time. This westwards movement of the magnetic field pattern at the Earth's surface, particularly prevalent in the Atlantic hemisphere, is related to the motion of fluid at the core surface slowly westwards, dragging with it the magnetic field lines.

Using IGRF-11 to compute the root mean square magnetic field vector at the Earth's surface arising from all spherical harmonic terms $(n \leq 10)$, the centred dipole terms $(n=1)$ and the non-dipole terms $(1<n \leq 10)$, it can be seen in Figure 10 that since 1900, the geomagnetic field is weakening overall by becoming less dipolar. However the non-dipolar part is strengthening. This may have consequences for the trajectories of energetic charged particles that enter the Earth's magnetosphere. One manifestation of this is the deepening, and westwards movement, of the South Atlantic Anomaly, the region where the geomagnetic field is weaker than elsewhere (see Figures $\underline{2}$ and 9 ). Energetic charged particles are able to penetrate closer to the Earth and cause a radiation hazard for satellites passing through this region.

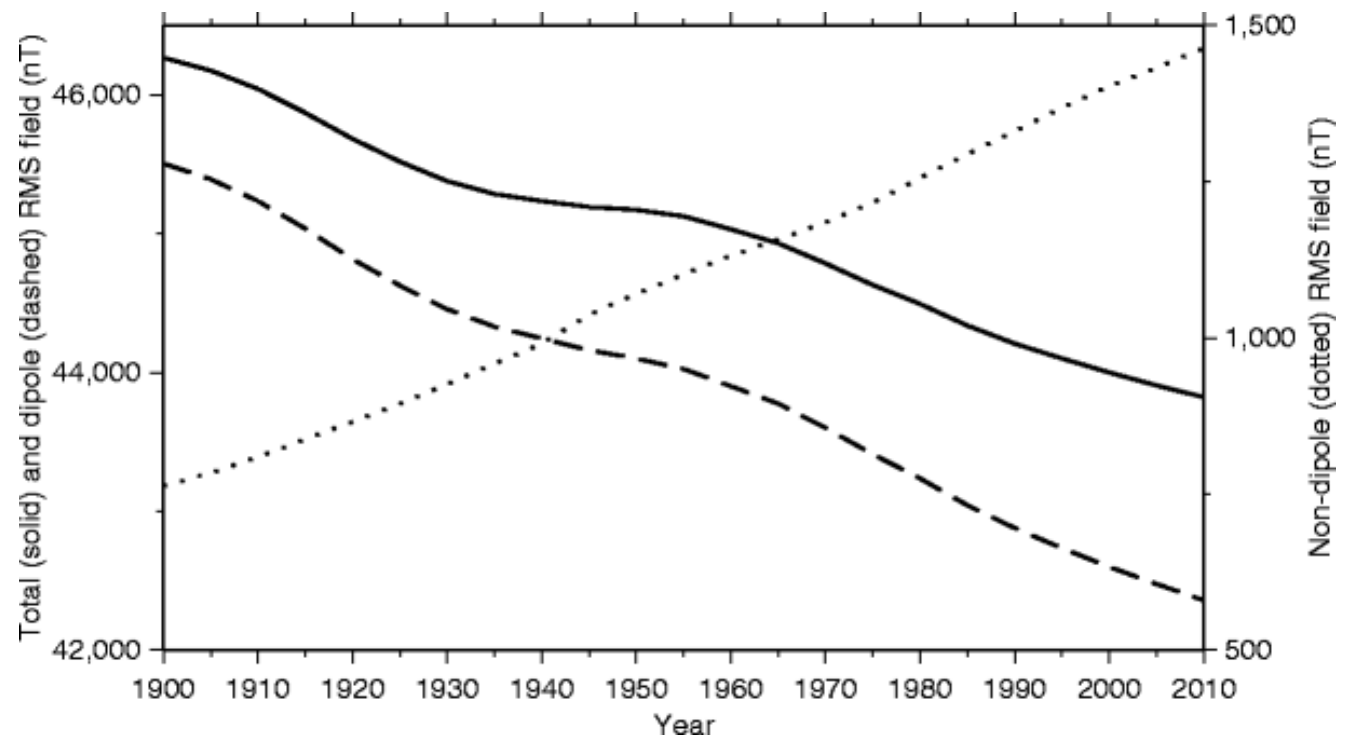


Geomagnetic Field, Global Pattern, Figure 10 The decline of the whole, and dipolar part of the geomagnetic field at the Earth's surface and the growth of the non-dipolar part since 1900, computed from IGRF-11.

Superimposed on these gradual changes of the magnetic field are the so-called geomagnetic jerks. They are relatively abrupt (duration of months) changes in the second time derivative, or secular acceleration, of the magnetic field. The first observed geomagnetic jerk was that around 1969, and since the late nineteenth century when direct and continuous measurements of the Earth's magnetic field have been available, geomagnetic jerks have also been observed to occur around 1925, 1978, 1991, and 1999. These jerks are most readily observed in the first time derivative of the easterly intensity at European observatories (Figure 11).

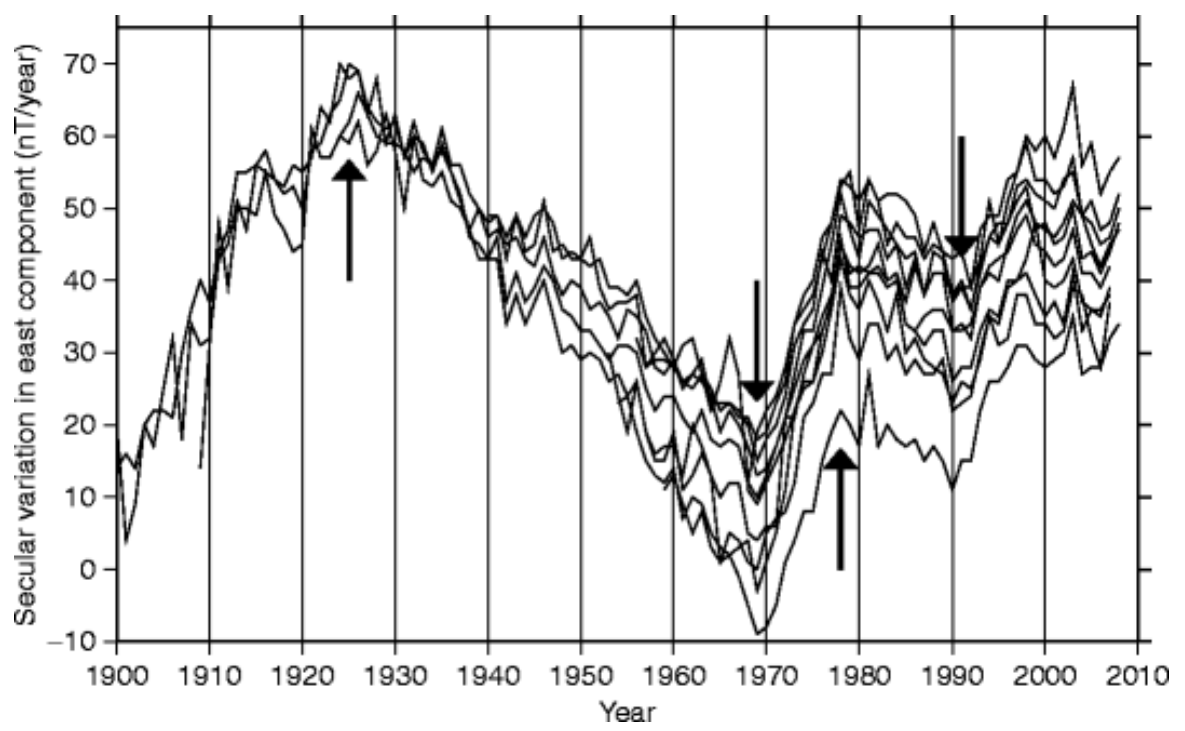

Geomagnetic Field, Global Pattern, Figure $\mathbf{1 1}$ Geomagnetic jerks as seen in the secular variation of the east component of the geomagnetic field observed at European observatories (IAGA codes of the observatories used are LRV, DOB, NUR, LER, ESK, HAD, DOU, BDV, FUR, CLF). Times of jerks are shown by arrows.

Various analysis techniques have been applied to jerks to investigate specific aspects of their temporal and spatial characteristics. Using wavelets, the 1969 and 1978 jerks have been shown to have different arrival times at the Earth's surface, with the northern hemisphere leading the southern hemisphere by about 2 years (Alexandrescu et al., 1996).

Understanding their origin is important, not only because they result from interesting dynamical processes in the core and may help determine the conductivity of the mantle, but also for improving time-dependent models of the geomagnetic field and for the strictly practical purpose of forecasting its future behaviour, for example, as used in navigation.

\section{Cross-references}

\section{Geomagnetic Field, IGRF}

\section{Geomagnetic Field, Measurement Techniques}

Geomagnetic Field, Secular Variation

Geomagnetic Field, Theory

Magnetic Methods, Satellite

Magnetic, Global Anomaly Map

\section{Bibliography}

Alexandrescu, M., Gibert, D., Hulot, G., Le Mouël, J.-L., and Saracco, G., 1996. Worldwide wavelet analysis of geomagnetic jerks. Journal of Geophysical Research, 101(B10), 21,975-21,994.

Barraclough, D. R., 1978. Spherical harmonic models of the geomagnetic field. Geomagnetic Bulletin Institute of Geological Sciences, No. 8. 


\begin{abstract}
Clark, T., and Barraclough, D., 2001. The first magnetic chart. Astronomy and Geophysics, 42, 1.23-1.25. Jackson, A., Art, R. T. J., and Matthew, R. W., 2000. Four centuries of geomagnetic secular variation from historical records.
Philosophical Transactions of the Royal Society of London Series A-Mathematical Physical and Engineering Sciences, 358, Jackson, A., Art, R. T. J., and Matthew, R. W., 2000. Four centuries of geomagnetic secular variation from historical records.
Philosophical Transactions of the Royal Society of London Series A-Mathematical Physical and Engineering Sciences, 358, 957-990.

Macmillan, S., and Finlay C., 2011. The International Geomagnetic Reference Field. In Mandea, M., Korte, M. (eds.), Geomagnetic Observations and Models. IAGA Special Sopron Book Series 5, DOI 10.1007/978-90-481-9858-0_10.

Malin, S. R. C., 1971. British World Magnetic Charts. In Zmuda, A. J. (ed.), World Magnetic Survey 1957-1969, IAGA Bulletin No. 28.

957-990.
\end{abstract}

(1)

